\title{
LIGHTLIKE SUBMERSIONS FROM TOTALLY UMBILICAL SEMI-TRANSVERSAL LIGHTLIKE SUBMANIFOLDS
}

\author{
RUPALI KAUSHAL, RAKESH KUMAR, AND RAKESH KUMAR NAGAICH
}

\author{
Received 21 December, 2017
}

\begin{abstract}
We study lightlike submersions from a totally umbilical semi-transversal lightlike submanifold of an indefinite Kaehler manifold onto an indefinite almost Hermitian manifold. We show that if an indefinite almost Hermitian manifold $B$ admits a lightlike submersion $\phi: M \rightarrow B$ from a totally umbilical semi-transversal lightlike submanifold $M$ of an indefinite Kaehler manifold $\bar{M}$ then $B$ is necessarily an indefinite Kaehler manifold. We investigate the condition for a totally umbilical semi-transversal lightlike submanifold $M$ to becomes a product manifold and its fibers become geodesic. Finally, we obtain some characterization theorems related to the sectional curvature of an indefinite Kaehler manifold.
\end{abstract}

2010 Mathematics Subject Classification: 53C20; 53C50

Keywords: indefinite Kaehler manifold, semi-transversal lightlike submanifolds, lightlike submersions

\section{INTRODUCTION}

The study of Riemannian submersions $\phi: M \rightarrow B$, from a Riemannian manifold $M$ onto a Riemannian manifold $B$ was initiated by O'Neill [10]. A Riemannian submersion naturally yields a vertical distribution, which is always integrable and a horizontal distribution. On the other hand, for a $C R$-submanifold $M$ of a Kaehler manifold $\bar{M}$ there are two orthogonal complementary distributions $D$ and $D^{\perp}$, such that $D$ is $\bar{J}$-invariant and $D^{\perp}$ is totally real and always integrable (cf. Bejancu [2]), where $\bar{J}$ is almost complex structure of $\bar{M}$. Kobayashi [9] observed the similarity between the total space of a Riemannian submersion and a $C R$-submanifold of a Kaehler manifold in terms of distributions. Then Kobayashi [9] introduced a submersion $\phi: M \rightarrow B$, from a $C R$-submanifold $M$ of a Kaehler manifold $\bar{M}$ onto an almost Hermitian manifold $B$ such that the distributions $D$ and $D^{\perp}$ of the $C R$-submanifold become the horizontal and the vertical distributions respectively, as required by the submersions and $\pi$ restricted to $D$ becomes a complex isometry.

Later, semi-Riemannian submersions were introduced by O'Neill in [11]. As it is known that when $M$ and $B$ are Riemannian manifolds then the fibers are always 
Riemannian manifolds. However, when the manifolds are semi-Riemannian manifolds then the fibers may not be Riemannian (hence semi-Riemannian) manifolds, (see [15]). Therefore in [13], Sahin introduced a screen lightlike submersion from a lightlike manifold onto a semi-Riemannian manifold and in [15], Sahin and Gunduzalp introduced a lightlike submersion from a semi-Riemannian manifold onto a lightlike manifold. It is well-known that semi-Riemannian submersions are of interest in mathematical physics, owing to their applications in the Yang-Mills theory, Kaluza-Klein theory, supergravity and superstring theories [3, 4, 8, 16]. Moreover, the geometry of lightlike submanifolds has potential for applications in mathematical physics, particularly in general relativity (for detail, see [5]) therefore in present paper, we study lightlike submersions from a totally umbilical semi-transversal lightlike submanifold of an indefinite Kaehler manifold onto an almost Hermitian manifold.

\section{LIGHTLIKE SUBMANIFOLDS}

Let $(\bar{M}, \bar{g})$ be a real $(m+n)$-dimensional semi-Riemannian manifold of constant index $q$ such that $m, n \geq 1,1 \leq q \leq m+n-1$ and $(M, g)$ be an $m$-dimensional submanifold of $\bar{M}$ and $g$ be the induced metric of $\bar{g}$ on $M$. If $\bar{g}$ is degenerate on the tangent bundle $T M$ of $M$ then $M$ is called a lightlike submanifold of $\bar{M}$, (see [5]). For a degenerate metric $g$ on $M, T M^{\perp}$ is a degenerate $n$-dimensional subspace of $T_{x} \bar{M}$. Thus both $T_{x} M$ and $T_{x} M^{\perp}$ are degenerate orthogonal subspaces but no longer complementary. In this case, there exists a subspace $\operatorname{Rad}\left(T_{x} M\right)=T_{x} M \cap T_{x} M^{\perp}$ which is known as radical (null) subspace. If the mapping $\operatorname{Rad}(T M): x \in M \longrightarrow$ $\operatorname{Rad}\left(T_{x} M\right)$, defines a smooth distribution on $M$ of rank $r>0$ then the submanifold $M$ of $\bar{M}$ is called an $r$-lightlike submanifold and $\operatorname{Rad}(T M)$ is called the radical distribution on $M$.

Screen distribution $S(T M)$ is a semi-Riemannian complementary distribution of $\operatorname{Rad}(T M)$ in $T M$, that is, $T M=\operatorname{Rad}(T M) \perp S(T M)$ and $S\left(T M^{\perp}\right)$ is a complementary vector subbundle to $\operatorname{Rad}(T M)$ in $T M^{\perp}$. Let $\operatorname{tr}(T M)$ and $\operatorname{ltr}(T M)$ be complementary (but not orthogonal) vector bundles to $T M$ in $\left.T \bar{M}\right|_{M}$ and to $\operatorname{Rad}(T M)$ in $S\left(T M^{\perp}\right)^{\perp}$ respectively. Then $\left.T \bar{M}\right|_{M}=T M \oplus \operatorname{tr}(T M)=$ $(\operatorname{Rad} T M \oplus \operatorname{ltr}(T M)) \perp S(T M) \perp S\left(T M^{\perp}\right)$.

Theorem 1 ([5]). Let $\left(M, g, S(T M), S\left(T M^{\perp}\right)\right)$ be an r-lightlike submanifold of a semi-Riemannian manifold $(\bar{M}, \bar{g})$. Then there exists a complementary vector bundle ltr $(T M)$ of $\operatorname{Rad}(T M)$ in $S\left(T M^{\perp}\right)^{\perp}$ and a basis of ltr $(T M) \mid u$ consisting of smooth section $\left\{N_{i}\right\}$ of $S\left(T M^{\perp}\right)^{\perp} \mid u$, where $U$ is a coordinate neighborhood of M such that

$$
\bar{g}\left(N_{i}, \xi_{j}\right)=\delta_{i j}, \quad \bar{g}\left(N_{i}, N_{j}\right)=0, \text { for any } \quad i, j \in\{1,2, . ., r\},
$$

where $\left\{\xi_{1}, \ldots, \xi_{r}\right\}$ is a lightlike basis of $\operatorname{Rad}(T M)$. 
Let $\bar{\nabla}$ be the Levi-Civita connection on $\bar{M}$ then for any $X, Y \in \Gamma(T M)$ and $U \in$ $\Gamma(\operatorname{tr}(T M))$, the Gauss and Weingarten formulas are given by

$$
\bar{\nabla}_{X} Y=\nabla_{X} Y+h(X, Y), \quad \bar{\nabla}_{X} U=-A_{U} X+\nabla_{X}^{\perp} U,
$$

where $\left\{\nabla_{X} Y, A_{U} X\right\}$ and $\left\{h(X, Y), \nabla_{X}^{\perp} U\right\}$ belong to $\Gamma(T M)$ and $\Gamma(\operatorname{tr}(T M))$, respectively. Here $\nabla$ is a torsion-free linear connection on $M, h$ is a symmetric bilinear form on $\Gamma(T M)$ which is called the second fundamental form, $A_{U}$ is a linear operator on $M$ and known as a shape operator.

Considering the projection morphisms $L$ and $S$ of $\operatorname{tr}(T M)$ on $\operatorname{ltr}(T M)$ and $S\left(T M^{\perp}\right)$, respectively, then (2.2) becomes

$$
\bar{\nabla}_{X} Y=\nabla_{X} Y+h^{l}(X, Y)+h^{s}(X, Y), \quad \bar{\nabla}_{X} U=-A_{U} X+D_{X}^{l} U+D_{X}^{s} U,
$$

where $h^{l}(X, Y)=L(h(X, Y)), h^{s}(X, Y)=S(h(X, Y)), D_{X}^{l} U=L\left(\nabla_{X}^{\perp} U\right), D_{X}^{s} U=$ $S\left(\nabla_{X}^{\perp} U\right)$. As $h^{l}$ and $h^{s}$ are $\operatorname{ltr}(T M)$-valued and $S\left(T M^{\perp}\right)$-valued respectively, therefore they are called as the lightlike second fundamental form and the screen second fundamental form on $M$. In particular

$$
\bar{\nabla}_{X} N=-A_{N} X+\nabla_{X}^{l} N+D^{s}(X, N), \quad \bar{\nabla}_{X} W=-A_{W} X+\nabla_{X}^{s} W+D^{l}(X, W),
$$

where $X \in \Gamma(T M), N \in \Gamma(\operatorname{lt} r(T M))$ and $W \in \Gamma\left(S\left(T M^{\perp}\right)\right)$. Using (2.3) and (2.4), we obtain

$$
\bar{g}\left(h^{S}(X, Y), W\right)+\bar{g}\left(Y, D^{l}(X, W)\right)=g\left(A_{W} X, Y\right) .
$$

Let $\bar{R}$ and $R$ be the curvature tensors of $\bar{\nabla}$ and $\nabla$, respectively then by straightforward calculations (see [5]), we have

$$
\begin{aligned}
& \bar{R}(X, Y) Z=R(X, Y) Z+A_{h^{l}(X, Z)} Y-A_{h^{l}(Y, Z)} X+A_{h^{s}(X, Z)} Y \\
& -A_{h^{s}(Y, Z)} X+\left(\nabla_{X} h^{l}\right)(Y, Z)-\left(\nabla_{Y} h^{l}\right)(X, Z) \\
& +D^{l}\left(X, h^{s}(Y, Z)\right)-D^{l}\left(Y, h^{s}(X, Z)\right)+\left(\nabla_{X} h^{s}\right)(Y, Z) \\
& -\left(\nabla_{Y} h^{s}\right)(X, Z)+D^{s}\left(X, h^{l}(Y, Z)\right)-D^{s}\left(Y, h^{l}(X, Z)\right) \text {. }
\end{aligned}
$$

\section{SEMI-TRANSVERSAL LIGHTLIKE SUBMANIFOLDS}

Let $(\bar{M}, \bar{J}, \bar{g})$ be an indefinite almost Hermitian manifold and $\bar{\nabla}$ be the Levi-Civita connection on $\bar{M}$ with respect to the indefinite metric $\bar{g}$. Then $\bar{M}$ is called an indefinite Kaehler manifold [1] if the almost complex structure $\bar{J}$ is parallel with respect to $\bar{\nabla}$, that is $\left(\bar{\nabla}_{X} \bar{J}\right) Y=0$, for any $X, Y \in \Gamma(T \bar{M})$.

Definition 1 ([12]). Let $M$ be a lightlike submanifold of an indefinite Kaehler manifold $\bar{M}$ then $M$ is called a semi-transversal lightlike submanifold of $\bar{M}$ if the following conditions are satisfied:

(i) $\operatorname{Rad}(T M)$ is transversal with respect to $\bar{J}$. 
(ii) There exists a real non-null distribution $D \subset S(T M)$ such that $S(T M)=$ $D \oplus D^{\perp}, \bar{J}(D)=D, \bar{J} D^{\perp} \subset S\left(T M^{\perp}\right)$, where $D^{\perp}$ is orthogonal complementary to $D$ in $S(T M)$.

Then tangent bundle of a semi-transversal lightlike submanifold is decomposed as $T M=D \perp D^{\prime}$, where $D^{\prime}=D^{\perp} \perp \operatorname{Rad}(T M)$. We say $M$ is a proper semi-transversal lightlike submanifold if $D \neq\{0\}$ and $D^{\perp} \neq\{0\}$. Therefore $\operatorname{dim}(\operatorname{Rad}(T M)) \geq 2$ and for a proper $M, \operatorname{dim}(D) \geq 2 s, s>1, \operatorname{dim}\left(D^{\perp}\right) \geq 1$ and $\operatorname{dim}(\operatorname{Rad}(T M))=$ $\operatorname{dim}(\operatorname{ltr}(T M))$. Thus $\operatorname{dim}(M) \geq 5$ and $\operatorname{dim}(\bar{M}) \geq 8$. Next, we give example of semi-transversal lightlike submanifolds.

Example 1. Let $M$ be a 5-dimensional submanifold of $\left(R_{2}^{10}, \bar{g}\right)$ given by $x_{1}=$ $u_{1} \cosh \theta, x_{2}=u_{2} \cosh \theta, x_{3}=u_{1} \sinh \theta, x_{4}=u_{2} \sinh \theta, x_{5}=u_{3}, x_{6}=\sqrt{1-u_{3}^{2}}$, $x_{7}=u_{4}, \quad x_{8}=u_{8}, \quad x_{9}=u_{2}, \quad x_{10}=u_{1}, \quad$ where $\bar{g}$ is of signature $(-,-,+,+,+,+,+,+,+,+)$ with respect to the canonical basis $\left\{\partial x_{1}, \partial x_{2}, \partial x_{3}, \partial x_{4}, \partial x_{5}, \partial x_{6}, \partial x_{7}, \partial x_{8}, \partial x_{9}, \partial x_{10}\right\}$. Then $T M$ is spanned by $Z_{1}=$ $\cosh \theta \partial x_{1}+\sinh \theta \partial x_{3}+\partial x_{10}, Z_{2}=\cosh \theta \partial x_{2}+\sinh \theta \partial x_{4}+\partial x_{9}, Z_{3}=x_{6} \partial x_{5}-$ $x_{5} \partial x_{6}, Z_{4}=\partial x_{7}, Z_{5}=\partial x_{8}$. Clearly $M$ is a 2-lightlike submanifold with $\operatorname{Rad}(T M)$ $=\operatorname{span}\left\{Z_{1}, Z_{2}\right\}$ and the lightlike transversal bundle is spanned by

$N_{1}=\frac{1}{2}\left(-\cosh \theta \partial x_{1}-\sinh \theta \partial x_{3}+\partial x_{10}\right), N_{2}=-\frac{1}{2}\left(\cosh \theta \partial x_{2}+\sinh \theta \partial x_{4}-\partial x_{9}\right)$, and $\bar{J} Z_{1}=-2 N_{2}$ and $\bar{J} Z_{2}=2 N_{1}$. Hence $\bar{J}(\operatorname{Rad}(T M))=\operatorname{ltr}(T M)$. Since $\bar{J} Z_{4}=Z_{5}$ then $D=\operatorname{span}\left\{Z_{4}, Z_{5}\right\}$ which is an invariant distribution on $M$. By direct calculations, the transversal screen bundle $S\left(T M^{\perp}\right)$ ) is spanned by

$W_{1}=\sinh \theta \partial x_{1}+\cosh \theta \partial x_{3}, \quad W_{2}=\sinh \theta \partial x_{2}+\cosh \theta \partial x_{4}, \quad W_{3}=x_{6} \partial x_{6}+x_{5} \partial x_{5}$. Thus $\bar{J} W_{3}=-Z_{3}$. Hence $D^{\perp}=\operatorname{span}\left\{Z_{3}\right\}$ is an anti-invariant distribution on $M$ and $\operatorname{span}\left\{W_{1}, W_{2}\right\}$ is invariant and $\operatorname{span}\left\{W_{3}\right\}$ is anti-invariant subbundles of $S\left(T M^{\perp}\right)$ respectively. Thus it enables us to choose $S(T M)=\operatorname{span}\left\{Z_{3}, Z_{4}, Z_{5}\right\}$. Hence $M$ is a proper semi-transversal lightlike submanifold.

Let $M$ be a semi-transversal lightlike submanifold of an indefinite Kaehler manifold $\bar{M}$. Let $Q, P_{1}, P_{2}$ and $P$ be the projection morphisms from $T M$ on $D$, $\operatorname{Rad}(T M), D^{\perp}$ and $D^{\prime}$ respectively. Then for any $X \in \Gamma(T M)$, we put

$$
X=Q X+P_{1} X+P_{2} X .
$$

Applying $\bar{J}$ to (3.1), we obtain $\bar{J} X=\bar{J} Q X+\bar{J} P_{1} X+\bar{J} P_{2} X$, can be written as $\bar{J} X=T Q X+w P_{1} X+w P_{2} X$. Put $w P_{1}=w_{1}$ and $w P_{2}=w_{2}$, then we have

$$
\bar{J} X=T X+w_{1} X+w_{2} X,
$$

where $T X \in \Gamma(D), w_{1} X \in \Gamma(\operatorname{ltr}(T M))$ and $w_{2} X \in \Gamma\left(\bar{J} D^{\perp}\right) \subset S\left(T M^{\perp}\right)$. Similarly, for any $V \in \Gamma\left(S\left(T M^{\perp}\right)\right)$, we can write

$$
\bar{J} V=E V+F V
$$


where $E V \in \Gamma\left(D^{\perp}\right)$ and $F V \in \Gamma(\mu)$, where $\mu$ is a complementary bundle of $\bar{J} D^{\perp}$ in $S\left(T M^{\perp}\right.$ ). Differentiating (3.2) and using (2.3), (2.4) and (3.3), for any $X \in$ $\Gamma(T M)$, we have the following lemma.

Lemma 1. Let $M$ be a semi-transversal lightlike submanifold of an indefinite Kaehler manifold $\bar{M}$. Then we have

$$
\begin{gathered}
\left(\nabla_{X} T\right) Y=A_{w_{1} Y} X+A_{w_{2} Y} X+\bar{J} h^{l}(X, Y)+E h^{s}(X, Y), \\
\left(\nabla_{X} w_{1}\right) Y=-h^{l}(X, T Y)-D^{l}\left(X, w_{2} Y\right), \\
\left(\nabla_{X} w_{2}\right) Y=F h^{s}(X, Y)-h^{s}(X, T Y)-D^{s}\left(X, w_{1} Y\right), \text { where } \\
\left(\nabla_{X} T\right) Y=\nabla_{X} T Y-T \nabla_{X} Y, \quad\left(\nabla_{X} w_{1}\right) Y=\nabla_{X}^{l} w_{1} Y-w_{1} \nabla_{X} Y, \\
\left(\nabla_{X} w_{2}\right) Y=\nabla_{X}^{s} w_{2} Y-w_{2} \nabla_{X} Y .
\end{gathered}
$$

Definition 2 ([6]). A lightlike submanifold $(M, g)$ of a semi-Riemannian manifold $(\bar{M}, \bar{g})$ is said to be a totally umbilical in $\bar{M}$ if there is a smooth transversal vector field $H \in \Gamma(\operatorname{tr}(T M))$ on $M$, called the transversal curvature vector field of $M$, such that $h(X, Y)=H \bar{g}(X, Y)$, for $X, Y \in \Gamma(T M)$. Using (2.3), clearly $M$ is a totally umbilical, if and only if, for $X, Y \in \Gamma(T M)$ and $W \in \Gamma\left(S\left(T M^{\perp}\right)\right)$, on each coordinate neighborhood $\mathcal{U}$ there exist smooth vector fields $H^{l} \in \Gamma(\operatorname{ltr}(T M))$ and $H^{s} \in \Gamma\left(S\left(T M^{\perp}\right)\right)$ such that

$$
h^{l}(X, Y)=H^{l} g(X, Y), \quad h^{s}(X, Y)=H^{s} g(X, Y), \quad D^{l}(X, W)=0 .
$$

Lemma 2. Let $M$ be a totally umbilical semi-transversal lightlike submanifold of an indefinite Kaehler manifold $\bar{M}$ then the distribution $D^{\prime}$ defines a totally geodesic foliation in $M$.

Proof. Let $X, Y \in \Gamma\left(D^{\prime}\right)$ then using (3.4) and (3.7), we obtain $T \nabla_{X} Y=$ $-A_{w_{1} Y} X-A_{w_{2} Y} X-\bar{J} h^{l}(X, Y)-E h^{s}(X, Y)$. On taking inner product both sides with $Z \in \Gamma(D)$, we further obtain

$$
\begin{aligned}
g\left(T \nabla_{X} Y, Z\right) & =\bar{g}\left(\bar{\nabla}_{X} w_{1} Y, Z\right)+\bar{g}\left(\bar{\nabla}_{X} w_{2} Y, Z\right)=-\bar{g}\left(\bar{J} Y, \bar{\nabla}_{X} Z\right) \\
& =\bar{g}\left(Y, \bar{\nabla}_{X} \bar{J} Z\right)=g\left(Y, \nabla_{X} Z^{\prime}\right),
\end{aligned}
$$

where $Z^{\prime}=\bar{J} Z \in \Gamma(D)$. Since $M$ is a totally umbilical lightlike submanifold then for any $X \in \Gamma\left(D^{\prime}\right)$ and $Z \in \Gamma(D)$, with (3.5) and (3.7), we have $w_{1} \nabla_{X} Z=$ $h^{l}(X, T Z)=H^{l} g(X, T Z)=0$ and using (3.6) and (3.8), we have $w_{2} \nabla_{X} Z=$ $-F h^{s}(X, Z)+h^{s}(X, T Z)=-F H^{s} g(X, Z)+H^{s} g(X, T Z)=0$, these facts imply that $\nabla_{X} Z \in \Gamma(D)$, for any $X \in \Gamma\left(D^{\prime}\right)$ and $Z \in \Gamma(D)$. Therefore (3.10) implies that $g\left(T \nabla_{X} Y, Z\right)=0$, then the non degeneracy of the distribution $D$ implies that $T \nabla_{X} Y=0$. Hence the result follows.

Theorem 2 ([12]). Let $M$ be a semi-transversal lightlike submanifold of an indefinite Kaehler manifold $\bar{M}$. Then the distribution $D^{\prime}$ is integrable, if and only if $A_{w Z} V=A_{w V} Z$, for any $Z, V \in \Gamma\left(D^{\prime}\right)$. 
Theorem 3. Let $M$ be a totally umbilical semi-transversal lightlike submanifold of an indefinite Kaehler manifold $\bar{M}$ then the distribution $D^{\prime}$ is integrable.

Proof. Let $X, Y \in \Gamma\left(D^{\prime}\right)$ then using (3.4) and (3.7) with the Lemma 2, we get $A_{w Y} X=-\bar{J} h^{l}(X, Y)-E h^{s}(X, Y)$ this implies that $A_{w Y} X \in \Gamma\left(D^{\prime}\right)$ and moreover the symmetric property of the second fundamental form $h$ gives that $A_{w Y} X=A_{w X} Y$. Hence by virtue of the Theorem 2, the result follows.

\section{SEMI-TRANSVERSAL LIGHTLIKE SUBMERSIONS}

Let $\phi: M \rightarrow B$ be a mapping from a Riemannian manifold $M$ onto a Riemannian manifold $B$ then it is said to be a Riemannian submersion if it satisfies the following axioms:

A1. $\phi$ has maximal rank. This implies that for each $b \in B, \phi^{-1}(b)$ is a submanifold of $M$, known as fiber, of dimension $\operatorname{dim} M-\operatorname{dim} B$. A vector field tangent to the fibers is called vertical vector field and orthogonal to fibers is called horizontal vector field.

A2. $\phi_{*}$ preserves the lengths of horizontal vectors.

The Riemannian submersions were introduced by O'Neill in [10] and since then plenty of work on this subject matter has been done (for detail, see [7,14] and many references therein). In the study of submersions, the vertical distribution $\mathcal{V}$ of $M$ is defined by $\mathcal{V}_{p}=k e r d \phi_{p}, p \in M$, which is always integrable and the orthogonal complementary distribution to $\mathcal{V}$ is defined by $\mathscr{H}_{p}=\left(\text { ker } d \phi_{p}\right)^{\perp}$, denoted by $\mathscr{H}$ and called a horizontal distribution. Therefore the tangent bundle $T M$ of $M$ has the following decomposition $T M=\mathcal{V} \oplus \mathscr{H}$.

Since the vertical distribution of the Riemannian submersion $\phi: M \rightarrow B$ and the totally real distribution $D^{\perp}$ of the $C R$-submanifold $M$ of a Kaehler manifold are always integrable. Therefore Kobayashi [9] introduced the submersion $\phi: M \rightarrow B$ from a $C R$-submanifold $M$ of a Kaehler manifold onto an almost Hermitian manifold $B$ such that the distributions $D$ and $D^{\perp}$ of the $C R$-submanifold become the horizontal and the vertical distributions respectively, required by the submersion and $\phi$ restricted to $D$ becomes a complex isometry.

We have seen that for a Riemannian submersion, the tangent bundle of the source manifold splits into horizontal and vertical part. On the other hand, the tangent bundle of a lightlike submanifold splits into screen and radical part and these natural splitting of the tangent bundle plays an important role in the study of lightlike submanifolds. Therefore Sahin [13] introduced screen lightlike submersion between a lightlike manifold and a semi-Riemannian manifold. Further in [15], Sahin and Gunduzalp introduced the idea of a lightlike submersion from a semi-Riemannian manifold onto a lightlike manifold.

From Theorem 3, we know that for a totally umbilical semi-transversal lightlike submanifold of an indefinite Kaehler manifold the distribution $D^{\prime}$ is integrable. Then 
a totally umbilical semi-transversal lightlike submanifold meets our requirements to define a submersion on it analogous to a submersion of a $C R$-submanifold. Significant applications of semi-Riemannian submersions in physics and the growing importance of lightlike submanifolds and hypersurfaces in mathematical physics, especially in relativity (see [5]), motivated us to work on this subject matter.

Definition 3. Let $\left(M, g_{M}, D\right)$ be a totally umbilical semi-transversal lightlike submanifold of an indefinite Kaehler manifold $\bar{M}$ and $\left(B, g_{B}\right)$ be an indefinite almost Hermitian manifold. Then we say that a smooth mapping $\phi:\left(M, g_{M}, D\right) \rightarrow\left(B, g_{B}\right)$ is a lightlike submersion if

(a) at every $p \in M, \mathcal{V}_{p}=\operatorname{ker}(d \phi)_{p}=D^{\prime}$.

(b) at each point $p \in M$, the differential $d \phi_{p}$ restricts to an isometry of the horizontal space $\mathscr{H}_{p}=D_{p}$ onto $T_{\phi(p)} B$, that is, $g_{D}(X, Y)=g_{B}(d \phi(X), d \phi(Y))$, for every vector fields $X, Y \in \Gamma(D)$.

Obviously from the definition, the restriction of the differential $d \phi_{p}$ to the distribution $\mathscr{H}_{p}=D_{p}$ maps that space isomorphically onto $T_{\phi(p)} B$. Then for any tangent vector $\widetilde{X} \in T_{\phi(p)} B$, we say that the tangent vector $X \in D_{p}$ is a horizontal lift of $\widetilde{X}$ as for submersions. If $\widetilde{X}$ is a vector field on an open subset $U$ of $B$ then the horizontal lift of $\widetilde{X}$ is the vector field $X \in \Gamma(D)$ on $\phi^{-1}(U)$ such that $d \phi(X)=\widetilde{X} o \phi$ and the vector field $X$ is called a basic vector field. Now, we give example of lightlike submersions.

Example 2. Let $M$ be a 5-dimensional semi-transversal lightlike submanifold of $R_{2}^{10}$ as in Example (1) and $B=R_{1}^{2}$ be an indefinite almost Hermitian manifold. Let the metrics be defined as $g_{M}=-\left(d x_{1}\right)^{2}-\left(d x_{2}\right)^{2}+\left(d x_{3}\right)^{2}+\left(d x_{4}\right)^{2}+\left(d x_{5}\right)^{2}$ and $g_{B}=-\left(d y_{1}\right)^{2}+\left(d y_{2}\right)^{2}$, where $x_{1}, x_{2}, x_{3}, x_{4}, x_{5}, x_{6}, x_{7}, x_{8}, x_{9}, x_{10}$ and $y_{1}, y_{2}$ be the canonical co-ordinates of $R_{2}^{10}$ and $R^{2}$, respectively. We define a map $\phi$ : $\left(x_{1}, x_{2}, x_{3}, x_{4}, x_{5}, x_{6}, x_{7}, x_{8}, x_{9}, x_{10}\right) \in R_{2}^{10} \mapsto\left(x_{7}, x_{8}\right) \in R_{1}^{2}$. Then the kernel of $d \phi$ is

$$
\begin{aligned}
k e r(d \phi)=D^{\prime}=\operatorname{span}\left\{Z_{1}=\cosh \theta \partial x_{1}+\sinh \theta \partial x_{3}+\partial x_{10},\right. & \\
& \left.Z_{2}=\cosh \theta \partial x_{2}+\sinh \theta \partial x_{4}+\partial x_{9}, Z_{3}=x_{6} \partial x_{5}-x_{5} \partial x_{6}\right\},
\end{aligned}
$$

where $d \phi\left(Z_{1}\right)=0, d \phi\left(Z_{2}\right)=0$ and $d \phi\left(Z_{3}\right)=0$. By direct computation, we obtain $D=\operatorname{span}\left\{Z_{4}=\partial x_{7}, Z_{5}=\partial x_{8}\right\}$, where $d \phi\left(Z_{4}\right)=\partial y_{1}, d \phi\left(Z_{5}\right)=\partial y_{2}$. Then it follows that $g_{M}\left(Z_{4}, Z_{4}\right)=g_{B}\left(d \phi\left(Z_{4}\right), d \phi\left(Z_{4}\right)\right)=1$ and $g_{M}\left(Z_{5}, Z_{5}\right)=$ $g_{B}\left(d \phi\left(Z_{5}\right), d \phi\left(Z_{5}\right)\right)=-1$. Hence $\phi$ is a semi-transversal lightlike submersion.

Theorem 4. Let $\phi: M \rightarrow B$ be a lightlike submersion from a totally umbilical semi-transversal lightlike submanifold of an indefinite Kaehler manifold $\bar{M}$ onto an indefinite almost Hermitian manifold $B$. If $X$ and $Y$ are basic vectors $\phi$-related to $\widetilde{X}, \widetilde{Y}$ respectively, then

(i) $g_{M}(X, Y)=g_{B}(\widetilde{X}, \widetilde{Y}) o \phi$. 
(ii) $[X, Y]^{\mathscr{H}}$ is the basic vector field and $\phi$-related to $[\widetilde{X}, \widetilde{Y}]$.

(iii) $\left(\nabla_{X}^{M} Y\right)^{\mathscr{H}}$ is the basic vector field and $\phi$-related to $\left(\nabla \frac{B}{\widetilde{Y}} \widetilde{Y}\right)$.

(iv) For any vertical vector field $V,[X, V]$ is vertical.

Proof. Let $X$ and $Y$ be basic vector fields of $M$ then ( $i$ ) follows immediately from part (b) of the Definition 3. Since $P$ and $Q$ be the projections from $T M$ on the distributions $D^{\prime}$ and $D$ of a semi-transversal lightlike submanifold of indefinite Kaehler manifold respectively, then $[X, Y]=P[X, Y]+Q[X, Y]$. Therefore the horizontal part $Q[X, Y]$ of $[X, Y]$ is a basic vector field and corresponds to $[\widetilde{X}, \widetilde{Y}]$, that is, $d \phi(Q[X, Y])=[d \phi(X), d \phi(Y)]$. Next, from the Koszul's formula, we have

$$
\begin{aligned}
2 g_{M}\left(\nabla_{X} Y, Z\right)= & X\left(g_{M}(Y, Z)\right)+Y\left(g_{M}(Z, X)\right)-Z\left(g_{M}(X, Y)\right) \\
& -g_{M}(X,[Y, Z])+g_{M}(Y,[Z, X])+g_{M}(Z,[X, Y])
\end{aligned}
$$

for any $X, Y, Z \in \Gamma(D)$. Consider $X, Y$ and $Z$ are the horizontal lifts of the vector fields $\widetilde{X}, \widetilde{Y}$ and $\widetilde{Z}$ respectively, then $X\left(g_{M}(Y, Z)\right)=\widetilde{X}\left(g_{B}(\widetilde{Y}, \widetilde{Z})\right) o \phi$ and $g_{M}(Z,[X, Y])=g_{B}(\widetilde{Z},[\widetilde{X}, \widetilde{Y}]) o \phi$ then from $(4.1)$, we have

$$
\begin{aligned}
2 g_{M}\left(\nabla_{X}^{M} Y, Z\right)= & \widetilde{X}\left(g_{B}(\widetilde{Y}, \widetilde{Z})\right) o \phi+\widetilde{Y}\left(g_{B}(\widetilde{Z}, \widetilde{X})\right) o \phi-\widetilde{Z}\left(g_{B}(\widetilde{X}, \widetilde{Y})\right) o \phi \\
& -g_{B}(\widetilde{X},[\widetilde{Y}, \widetilde{Z}]) o \phi+g_{B}(\widetilde{Y},[\widetilde{Z}, \widetilde{X}]) o \phi+g_{B}(\widetilde{Z},[\widetilde{X}, \widetilde{Y}]) o \phi \\
= & 2 g_{B}(\nabla \widetilde{X} \widetilde{Y}, \widetilde{Z}) .
\end{aligned}
$$

Thus from (4.2), (iii) follows, since $\phi$ is surjective and $\widetilde{Z}$ is arbitrarily chosen. Finally, let $V \in \Gamma\left(D^{\prime}\right)$ then $[X, V]$ is $\phi$-related to $[\widetilde{X}, 0]$, hence $(i v)$ follows and this completes the proof of the theorem.

Let $\nabla^{B}$ be the covariant differentiation on $B$ then we define the corresponding operator $\widetilde{\nabla}^{B}$ for basic vector fields of $B$ by assuming $\widetilde{\nabla}_{X}^{B} Y=\left(\nabla_{X}^{M} Y\right)^{\mathscr{H}}$, for any basic vector fields $X$ and $Y$. Thus from (iii) the Theorem $4, \widetilde{\nabla}_{X}^{B} Y$ is a basic vector

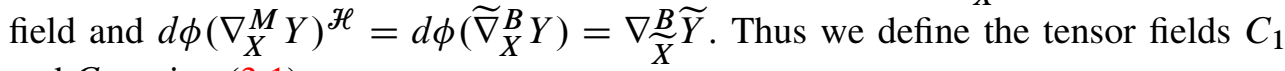
and $C_{2}$, using (3.1) as

$$
\nabla_{X}^{M} Y=\widetilde{\nabla}_{X}^{B} Y+C_{1}(X, Y)+C_{2}(X, Y)
$$

for any $X, Y \in \Gamma(D)$, where $C_{1}(X, Y)$ and $C_{2}(X, Y)$ denote the vertical parts of $\nabla_{X}^{M} Y$. It is easy to check that $C_{1}$ and $C_{2}$ are bilinear maps from $D \times D \rightarrow \operatorname{Rad}(T M)$ and $D \times D \rightarrow D^{\perp}$ respectively.

Theorem 5. Let $\phi: M \rightarrow B$ be a lightlike submersion of a totally umbilical semitransversal lightlike submanifold of an indefinite Kaehler manifold $\bar{M}$ onto an indefinite almost Hermitian manifold $B$ then for any basic vector fields $X$ and $Y$, we have

(i) the tensor fields $C_{1}$ and $C_{2}$ are skew-symmetric, that is, $C_{1}(X, Y)=$ $-C_{1}(Y, X)$ and $C_{2}(X, Y)=-C_{2}(Y, X)$; 
(ii) $P_{1}[X, Y]=2 C_{1}(X, Y)$ and $P_{2}[X, Y]=2 C_{2}(X, Y)$,

Proof. (i) Let $Z \in \Gamma\left(D^{\perp}\right)$ be any vertical vector field then for any basic vector field $X \in \Gamma(D)$, we have

$$
\begin{aligned}
0 & =Z(g(X, X))=2 \bar{g}\left(\bar{\nabla}_{Z} X, X\right)=2 g\left(\nabla_{X}^{M} Z-[X, Z], X\right)=-2 \bar{g}\left(Z, \bar{\nabla}_{X} X\right) \\
& =-2 g\left(Z, \widetilde{\nabla}_{X}^{B} X+C_{1}(X, X)+C_{2}(X, X)\right)=-2 g\left(Z, C_{2}(X, X)\right),
\end{aligned}
$$

then the non degeneracy of the distribution $D^{\perp}$ implies that $C_{2}(X, X)=0$, that is $C_{2}$ is skew-symmetric. Similarly, let $\bar{J} N \in \Gamma(\operatorname{Rad}(T M))$ be a vertical vector field where $N \in \Gamma(\operatorname{ltr}(T M))$, we have

$$
\begin{aligned}
0 & =\bar{J} N(g(X, X))=-2 \bar{g}\left(\bar{\nabla}_{N} X, X\right)=-2 g\left(\nabla_{X}^{M} N-[X, N], X\right) \\
& =2 g\left(N, \widetilde{\nabla}_{X}^{B} X+C_{1}(X, X)+C_{2}(X, X)\right)=2 g\left(N, C_{1}(X, X)\right),
\end{aligned}
$$

then using (2.1), we obtain $C_{1}(X, X)=0$, that is $C_{1}$ is skew-symmetric.

(ii) For basic vector fields $X, Y \in \Gamma(D)$, we have $[X, Y]=\nabla_{X}^{M} Y-\nabla_{Y}^{M} X$, using (3.1), (4.3) and skew-symmetric property of $C_{1}$ and $C_{2}$, result follows.

Next for a basic vector field $X$ and a vertical vector field $Z$, using (3.1), we define the tensor field $T$ as

$$
\nabla_{X}^{M} Z=\left(\nabla_{X}^{M} Z\right)^{\mathscr{H}}+\left(\nabla_{X}^{M} Z\right)^{\mathcal{V}}=T_{X} Z+\left(\nabla_{X}^{M} Z\right)^{\mathcal{V}},
$$

where $T$ is a bilinear map from $D \times D^{\prime} \rightarrow D$. Since $[X, Z]=\nabla_{X}^{M} Z-\nabla_{Z}^{M} X$ and $[X, Z]$ is vertical therefore

$$
Q\left(\nabla_{X}^{M} Z\right)=Q\left(\nabla_{Z}^{M} X\right)=T_{X} Z, \quad\left(\nabla_{X}^{M} Z\right)^{\mathcal{V}}=\left(\nabla_{Z}^{M} X\right)^{\mathcal{V}} .
$$

Let $X$ and $Y$ be basic vector fields and $Z$ be a vertical vector field such that $Z \in$ $\Gamma\left(D^{\perp}\right)$ then using (4.3), the tensor fields $T$ and $C_{2}$ are related by

$$
g\left(T_{X} Z, Y\right)=\bar{g}\left(\bar{\nabla}_{X} Z, Y\right)=-g\left(Z, \nabla_{X} Y\right)=-g\left(Z, C_{2}(X, Y)\right),
$$

and if $Z \in \Gamma(\operatorname{Rad}(T M))$ then

$$
g\left(T_{X} Z, Y\right)=-\bar{g}\left(Z, h^{l}(X, Y)\right) .
$$

Theorem 6. Let $\phi: M \rightarrow B$ be a lightlike submersion of a totally umbilical semitransversal lightlike submanifold of an indefinite Kaehler manifold $\bar{M}$ onto an indefinite almost Hermitian manifold $B$ then $B$ is also an indefinite Kaehler manifold. Moreover if $\bar{H}$ and $H^{B}$ denote the holomorphic sectional curvatures of $\bar{M}$ and $B$, respectively then for any unit basic vector $X \in \Gamma(\mathcal{H})$ of $M$, we have

$$
\bar{R}^{\bar{M}}(X, \bar{J} X, X, \bar{J} X)=R^{B}(\widetilde{X}, \bar{J} \widetilde{X}, \widetilde{X}, \bar{J} \widetilde{X})+4\left\|H^{s}\right\|^{2} .
$$

Proof. Let $X, Y \in \Gamma(D)$ be basic vector fields then using (2.3) and (4.3), we have

$$
\bar{\nabla}_{X} Y=\widetilde{\nabla}_{X}^{B} Y+C_{1}(X, Y)+C_{2}(X, Y)+h^{l}(X, Y)+h^{s}(X, Y) .
$$


On applying $\bar{J}$ on both sides of (4.8), we obtain

$$
\begin{aligned}
\bar{J} \bar{\nabla}_{X} Y= & \bar{J} \widetilde{\nabla}_{X}^{B} Y+\bar{J} C_{1}(X, Y)+\bar{J} C_{2}(X, Y)+\bar{J} h^{l}(X, Y) \\
& +E h^{s}(X, Y)+F h^{s}(X, Y),
\end{aligned}
$$

on replacing $Y$ by $\bar{J} Y$ in (4.8), we have

$$
\bar{\nabla}_{X} \bar{J} Y=\widetilde{\nabla}_{X}^{B} \bar{J} Y+C_{1}(X, \bar{J} Y)+C_{2}(X, \bar{J} Y)+h^{l}(X, \bar{J} Y)+h^{s}(X, \bar{J} Y) .
$$

Since $\bar{M}$ is a Kaehler manifold therefore $\bar{\nabla}_{X} \bar{J} Y=\bar{J} \bar{\nabla}_{X} Y$, then equating (4.9) and (4.10), we obtain

$$
\begin{gathered}
\widetilde{\nabla}_{X}^{B} \bar{J} Y=\bar{J} \widetilde{\nabla}_{X}^{B} Y \in \Gamma(\mathcal{H}), \\
C_{1}(X, \bar{J} Y)=\bar{J} h^{l}(X, Y) \in \Gamma(\operatorname{Rad}(T M)), \\
C_{2}(X, \bar{J} Y)=E h^{s}(X, Y) \in \Gamma\left(D^{\perp}\right), \\
h^{s}(X, \bar{J} Y)=\bar{J} C_{2}(X, Y)+F h^{s}(X, Y) \in \Gamma\left(S\left(T M^{\perp}\right)\right), \\
h^{l}(X, \bar{J} Y)=\bar{J} C_{1}(X, Y) \in \Gamma(\operatorname{ltr}(T M)) .
\end{gathered}
$$

From (4.11), we see that almost complex structure $\bar{J}$ of $B$ is parallel and hence $B$ is also an indefinite Kaehler manifold.

From (3.3), it is clear that $U \in \Gamma\left(\bar{J} D^{\perp}\right) \subset S\left(T M^{\perp}\right)$, if and only if, $F U=0$ then $\bar{J} U=E U$ and $U \in \Gamma\left(\mu=\left(\bar{J} D^{\perp}\right)^{\perp}\right) \subset S\left(T M^{\perp}\right)$, if and only if, $E U=0$ then $\bar{J} U=F U$. Therefore from (4.13), (4.14) and skew-symmetric property of $C_{2}$, we obtain $C_{2}(X, \bar{J} Y)=C_{2}(Y, \bar{J} X), C_{2}(\bar{J} X, Y)=C_{2}(\bar{J} Y, X), C_{2}(\bar{J} X, \bar{J} Y)=$ $C_{2}(X, Y)$ and $h^{s}(X, \bar{J} Y)+h^{s}(Y, \bar{J} X)=2 F h^{s}(X, Y)$. On the other hand, since $M$ is a totally umbilical semi-transversal lightlike submanifold then we have $h^{s}(X, \bar{J} Y)+$ $h^{s}(Y, \bar{J} X)=g(X, \bar{J} Y) H^{s}+g(Y, \bar{J} X) H^{s}=0$. Therefore $F h^{s}(X, Y)=0$ and this implies that $h^{s}(X, Y) \in \Gamma\left(\bar{J} D^{\perp}\right)$, for any $X, Y \in \Gamma(D)$. By virtue of totally umbilical property of $M$, we also have $h^{s}(\bar{J} X, \bar{J} Y)=h^{s}(X, Y)$. Similarly using (4.12) and (4.15), we obtain $C_{1}(X, \bar{J} Y)=C_{1}(Y, \bar{J} X), C_{1}(\bar{J} X, Y)=C_{1}(\bar{J} Y, X), C_{1}(\bar{J} X, \bar{J} Y)$ $=C_{1}(X, Y)$ and $h^{l}(\bar{J} X, \bar{J} Y)=h^{l}(X, Y), h^{l}(\bar{J} X, Y)+h^{l}(X, \bar{J} Y)=0$. Now, for any $X, Y, Z \in \Gamma(D)$, using (4.3) and (4.4), we have

$$
\begin{gathered}
\nabla_{X} \nabla_{Y} Z=\widetilde{\nabla}_{X}^{B} \widetilde{\nabla}_{Y}^{B} Z+T_{X} C_{1}(Y, Z)+T_{X} C_{2}(Y, Z)+\text { vertical, } \\
\nabla_{Y} \nabla_{X} Z=\widetilde{\nabla}_{Y}^{B} \widetilde{\nabla}_{X}^{B} Z+T_{Y} C_{1}(X, Z)+T_{Y} C_{2}(X, Z)+\text { vertical, } \\
\nabla_{[X, Y]} Z=\widetilde{\nabla}_{Q[X, Y]}^{B} Z+2 T_{Z} C_{1}(X, Y)+2 T_{Z} C_{2}(X, Y)+\text { vertical. }
\end{gathered}
$$

Further using (4.16)-(4.18), we obtain

$$
\begin{aligned}
R^{M}(X, Y) Z= & \left(R^{B}(\widetilde{X}, \widetilde{Y}) \widetilde{Z}\right)^{*}+T_{X} C_{1}(Y, Z)+T_{X} C_{2}(Y, Z)-T_{Y} C_{1}(X, Z) \\
& -T_{Y} C_{2}(X, Z)-2 T_{Z} C_{1}(X, Y)-2 T_{Z} C_{2}(X, Y) \\
& + \text { vertical, }
\end{aligned}
$$


where $\left(R^{B}(\widetilde{X}, \widetilde{Y}) \widetilde{Z}\right)^{*}$ denotes the basic vector field of $M$ corresponding to $R^{B}(\widetilde{X}, \widetilde{Y}) \widetilde{Z}$. Using (4.19) in (2.6), we obtain

$$
\begin{aligned}
\bar{R}^{\bar{M}}(X, Y) Z= & \left(R^{B}(\widetilde{X}, \widetilde{Y}) \widetilde{Z}\right)^{*}+T_{X} C_{1}(Y, Z)+T_{X} C_{2}(Y, Z)-T_{Y} C_{1}(X, Z) \\
& -T_{Y} C_{2}(X, Z)-2 T_{Z} C_{1}(X, Y)-2 T_{Z} C_{2}(X, Y)+A_{h^{l}(X, Z)} Y \\
& -A_{h^{l}(Y, Z)} X+A_{h^{s}(X, Z)} Y-A_{h^{s}(Y, Z)} X+\left(\nabla_{X} h^{l}\right)(Y, Z) \\
& -\left(\nabla_{Y} h^{l}\right)(X, Z)+D^{l}\left(X, h^{s}(Y, Z)\right)-D^{l}\left(Y, h^{s}(X, Z)\right) \\
& +\left(\nabla_{X} h^{s}\right)(Y, Z)-\left(\nabla_{Y} h^{s}\right)(X, Z)+D^{s}\left(X, h^{l}(Y, Z)\right) \\
& -D^{s}\left(Y, h^{l}(X, Z)\right)+\text { vertical. }
\end{aligned}
$$

Now, for basic vector field $W \in \Gamma(D)$ with (2.4), (2.5), (4.4)-(4.7), we obtain

$$
\begin{aligned}
\bar{R}^{\bar{M}}(X, Y, Z, W)= & R^{B}(\widetilde{X}, \widetilde{Y}, \widetilde{Z}, \widetilde{W})-\bar{g}\left(C_{1}(Y, Z), h^{l}(X, W)\right) \\
& -g\left(C_{2}(Y, Z), C_{2}(X, W)\right)+\bar{g}\left(C_{1}(X, Z), h^{l}(Y, W)\right) \\
& +g\left(C_{2}(X, Z), C_{2}(Y, W)\right)+2 \bar{g}\left(C_{1}(X, Y), h^{l}(Z, W)\right) \\
& +2 g\left(C_{2}(X, Y), C_{2}(Z, W)\right)+g\left(A_{h^{l}(X, Z)} Y, W\right) \\
& -g\left(A_{h^{l}(Y, Z)} X, W\right)+\bar{g}\left(h^{s}(X, Z), h^{s}(Y, W)\right) \\
& -\bar{g}\left(h^{s}(Y, Z), h^{s}(X, W)\right) .
\end{aligned}
$$

Now, using (2.4) and (4.3), we have $g\left(A_{h^{l}(X, Z)} Y, W\right)=\bar{g}\left(h^{l}(X, Z), \bar{\nabla}_{Y} W\right)=$ $\bar{g}\left(h^{l}(X, Z), C_{1}(Y, W)\right)$ and similarly $g\left(A_{h^{l}(Y, Z)} X, W\right)=\bar{g}\left(h^{l}(Y, Z), C_{1}(X, W)\right)$. Using these expressions with (4.15) in (4.20), we obtain

$$
\begin{aligned}
\bar{R}^{\bar{M}}(X, Y, Z, W)= & R^{B}(\widetilde{X}, \widetilde{Y}, \widetilde{Z}, \widetilde{W})+\bar{g}\left(\bar{J} h^{l}(Y, \bar{J} Z), h^{l}(X, W)\right) \\
& -g\left(C_{2}(Y, Z), C_{2}(X, W)\right)-\bar{g}\left(\bar{J} h^{l}(X, \bar{J} Z), h^{l}(Y, W)\right) \\
& +g\left(C_{2}(X, Z), C_{2}(Y, W)\right)-2 \bar{g}\left(\bar{J} h^{l}(X, \bar{J} Y), h^{l}(Z, W)\right) \\
& +2 g\left(C_{2}(X, Y), C_{2}(Z, W)\right)-\bar{g}\left(\bar{J} h^{l}(Y, \bar{J} W), h^{l}(X, Z)\right) \\
& +\bar{g}\left(\bar{J} h^{l}(X, \bar{J} W), h^{l}(Y, Z)\right)+\bar{g}\left(h^{s}(X, Z), h^{s}(Y, W)\right) \\
& -\bar{g}\left(h^{s}(Y, Z), h^{s}(X, W)\right) .
\end{aligned}
$$

To compare holomorphic sectional curvature of $\bar{M}$ with that of $B$, set $Y=\bar{J} X$, $Z=X$ and $W=\bar{J} X$ in (4.21) and then using the hypothesis that $M$ is a totally umbilical semi-transversal lightlike submanifold, we obtain $\bar{R}^{\bar{M}}(X, \bar{J} X, X, \bar{J} X)=$ $R^{B}(\widetilde{X}, \bar{J} \widetilde{X}, \widetilde{X}, \bar{J} \widetilde{X})+\left\|C_{2}(X, X)\right\|^{2}+3\left\|C_{2}(X, \bar{J} X)\right\|^{2}+\left\|h^{s}(X, X)\right\|^{2}$. Since $F h^{s}(X, Y)=0$ therefore (4.14) implies $\left\|h^{s}(X, X)\right\|^{2}=\left\|C_{2}(X, \bar{J} X)\right\|^{2}$ and by virtue of the totally umbilical property of $M,(4.14)$ implies that $C_{2}(X, X)=-\bar{J} h^{s}(X, \bar{J} X)$ $=-\bar{J}\left(H^{S} g(X, \bar{J} X)\right)=0$. Thus the holomorphic sectional curvature of $\bar{M}$ is given 
as

$$
\begin{aligned}
\bar{R}^{\bar{M}}(X, \bar{J} X, X, \bar{J} X) & =R^{B}(\widetilde{X}, \bar{J} \widetilde{X}, \widetilde{X}, \bar{J} \widetilde{X})+4\left\|C_{2}(X, \bar{J} X)\right\|^{2} \\
& =R^{B}(\widetilde{X}, \bar{J} \widetilde{X}, \widetilde{X}, \bar{J} \widetilde{X})+4\left\|h^{s}(X, X)\right\|^{2} \\
& =R^{B}(\widetilde{X}, \bar{J} \widetilde{X}, \widetilde{X}, \bar{J} \widetilde{X})+4\left\|H^{s}\right\|^{2} .
\end{aligned}
$$

This completes the proof.

Theorem 7. Let $\phi: M \rightarrow B$ be a lightlike submersion of a totally umbilical semitransversal lightlike submanifold of an indefinite Kaehler manifold $\bar{M}$ onto an indefinite almost Hermitian manifold $B$. If the distribution $D$ is integrable, then $M$ is a lightlike product manifold.

Proof. Let the distribution $D$ be an integrable therefore $P_{1}[X, Y]=0$ and $P_{2}[X, Y]$ $=0$, for any $X, Y \in \Gamma(D)$, where $P_{1}$ and $P_{2}$ are the projection morphisms from $T M$ to $\operatorname{Rad}(T M)$ and $D^{\perp}$, respectively. Therefore using the Theorem 5, we have $C_{1}(X, Y)=0$ and $C_{2}(X, Y)=0$. Hence using (4.3), we obtain that $\nabla_{X}^{M} Y \in \Gamma(D)$, for any $X, Y \in \Gamma(D)$, consequently the distribution $D$ defines a totally geodesic foliation in $M$. Moreover, from the Lemma 2, the distribution $D^{\prime}$ also defines a totally geodesic foliation in $M$. Thus using the De Rham's theorem, $M$ is a product manifold $M_{1} \times M_{2}$, where $M_{1}$ and $M_{2}$ are the leaves of the distributions of $D$ and $D^{\prime}$.

Theorem 8. Let $\phi: M \rightarrow B$ be a lightlike submersion of a totally umbilical semitransversal lightlike submanifold of an indefinite Kaehler manifold $\bar{M}$ onto an indefinite almost Hermitian manifold B such that $\bar{J}\left(D^{\perp}\right)=S\left(T M^{\perp}\right)$. Then the fibers are totally geodesic submanifolds of $M$.

Proof. Let $U, V \in \Gamma\left(D^{\prime}\right)$ and then define

$$
\nabla_{U}^{M} V=\hat{\nabla}_{U} V+L(U, V),
$$

where $\hat{\nabla}_{U} V=\left(\nabla_{U}^{M} V\right)^{\mathcal{V}}$ and $L(U, V)=\left(\nabla_{U}^{M} V\right)^{\mathscr{H}}$. Since the distribution $D^{\prime}$ is integrable always, then $L(U, V)=L(V, U)$. Now, using the Kaehlerian property of $\bar{M}$, we have $\bar{\nabla}_{U} \bar{J} V=\bar{J} \bar{\nabla}_{U} V$, since $\bar{J}\left(D^{\perp}\right)=S\left(T M^{\perp}\right)$, then

$$
-A_{\bar{J} V} U+\nabla_{U}^{t} \bar{J} V=\bar{J} \hat{\nabla}_{U} V+\bar{J} L(U, V)+\bar{J} h(U, V) .
$$

On comparing the horizontal and vertical components both sides, we get

$$
\mathscr{H}\left(A_{\bar{J} V} U\right)=-\bar{J} L(U, V), \quad \mathcal{V}\left(A_{\bar{J} V} U\right)=-\bar{J} h(U, V) .
$$

From (4.22), it is clear that the fibers are totally geodesic submanifolds of $M$, if and only if, $L(U, V)=0$ or using (4.23) 1 , if and only if, $A_{\bar{J} V} U \in \Gamma\left(D^{\prime}\right)$, for any $U, V \in$ $\Gamma\left(D^{\prime}\right)$. Now, particularly choose $V \in D^{\perp}$ then using the hypothesis of this theorem $\bar{J} V \in \Gamma\left(S\left(T M^{\perp}\right)\right)$. Let $Y \in \Gamma(D)$ then using (2.5) with the fact that $\mathrm{M}$ is a totally umbilical lightlike submanifold, we obtain $g\left(A_{\bar{J} V} U, Y\right)=\bar{g}\left(h^{s}(U, Y), \bar{J} V\right)=g(U, Y) \bar{g}\left(H^{s}, \bar{J} V\right)=$ 0 . Similarly, let $V \in \Gamma(\operatorname{Rad}(T M))$ then $g\left(A_{\bar{J} V} U, Y\right)=\bar{g}\left(\bar{J} V, \bar{\nabla}_{U} Y\right)=-\bar{g}\left(V, h^{l}(U, \bar{J} Y)\right)=$ $-g(U, \bar{J} Y) \bar{g}\left(V, H^{l}\right)=0$. Thus $A_{\bar{J} V} U \in \Gamma\left(D^{\prime}\right)$ and the assertion follows. 
Theorem 9. Let $\phi: M \rightarrow B$ be a lightlike submersion of a totally umbilical semitransversal lightlike submanifold of an indefinite Kaehler manifold $\bar{M}$ onto an indefinite almost Hermitian manifold $B$. Then the sectional curvature of $\bar{M}$ and of the fiber are related by

$$
\begin{aligned}
\bar{K}(U \wedge V)= & \hat{K}(U \wedge V)+g\left(A_{h^{l}(U, U)} V, V\right)-g\left(A_{h^{l}(V, U)} U, V\right) \\
& +g\left(\left[A_{\bar{J} V}, A_{\bar{J} U}\right] U, V\right),
\end{aligned}
$$

for any orthonormal vector fields $U, V \in \Gamma\left(D^{\perp}\right)$.

Proof. Let $\nabla$ and $\hat{\nabla}$ be the connections of semi-transversal lightlike submanifold $M$ and its fiber, respectively. Let $R$ and $\hat{R}$ be the curvature tensors of $\nabla$ and $\hat{\nabla}$, respectively then for any $U, V \in \Gamma\left(D^{\perp}\right)$, using (4.22) we have

$$
\begin{aligned}
R(U, V) U= & \nabla_{U}\left(\hat{\nabla}_{V} U+L(V, U)\right)-\nabla_{V}\left(\hat{\nabla}_{U} U+L(U, U)\right) \\
& -\left(\hat{\nabla}_{[U, V]} U+L([U, V], U)\right),
\end{aligned}
$$

this further implies that

$$
\begin{aligned}
R(U, V, U, V)= & g\left(\nabla_{U} \hat{\nabla}_{V} U, V\right)+g\left(\nabla_{U} L(V, U), V\right)-g\left(\nabla_{V} \hat{\nabla}_{U} U, V\right) \\
& -g\left(\nabla_{V} L(U, U), V\right)-g\left(\hat{\nabla}_{[U, V]} U, V\right) .
\end{aligned}
$$

Again using (4.22), it leads to

$$
R(U, V, U, V)=\hat{R}(U, V, U, V)+g\left(\nabla_{U} L(V, U), V\right)-g\left(\nabla_{V} L(U, U), V\right) .
$$

Now, using the fact that $M$ is totally umbilical lightlike submanifold, we get

$$
\begin{aligned}
g\left(\nabla_{U} L(V, W), F\right) & =g\left(\bar{\nabla}_{U} L(V, W)-g\left(h^{l}(U, L(V, W)), F\right)\right. \\
& =-g\left(L(V, W), \nabla_{U} F\right)=-g(L(V, W), L(U, F)),
\end{aligned}
$$

for any $U, V, W, F \in \Gamma\left(D^{\perp}\right)$ therefore (4.24) becomes

$$
R(U, V, U, V)=\hat{R}(U, V, U, V)-g(L(U, V), L(U, V))+g(L(U, U), L(V, V)) .
$$

Using (2.5), (2.6) and $M$ is totally umbilical lightlike submanifold, we have

$$
\begin{aligned}
\bar{R}(U, V, U, V)= & R(U, V, U, V)+g\left(A_{h^{l}(U, U)} V, V\right)-g\left(A_{h^{l}(V, U)} U, V\right) \\
& +\bar{g}\left(h^{s}(V, V), h^{s}(U, U)\right)-\bar{g}\left(h^{s}(U, V), h^{s}(V, U)\right) .
\end{aligned}
$$

Further using (4.23), (4.25) and the fact $L(U, V)=L(V, U)$, we obtain

$$
\begin{aligned}
\bar{R}(U, V, U, V)= & \hat{R}(U, V, U, V)-g\left(\mathscr{H}\left(A_{\bar{J} U} V\right), \mathcal{H}\left(A_{\bar{J} U} V\right)\right) \\
& +g\left(\mathscr{H}\left(A_{\bar{J} U} U\right), \mathscr{H}\left(A_{\bar{J} V} V\right)\right)+g\left(A_{h^{l}(U, U)} V, V\right) \\
& -g\left(A_{h^{l}(V, U)} U, V\right)+g\left(\mathcal{V}\left(A_{\bar{J} V} V\right), \mathcal{V}\left(A_{\bar{J} U} U\right)\right) \\
& -g\left(\mathcal{V}\left(A_{\bar{J} V} U\right), \mathcal{V}\left(A_{\bar{J} V} U\right)\right) .
\end{aligned}
$$


Since $U, V \in \Gamma\left(D^{\perp}\right)$ and let $X \in \Gamma(D)$ then using (2.3), we get $g\left(A_{\bar{J} U} V, X\right)=0$, which further implies that $A_{\bar{J} U} V \in \Gamma\left(D^{\perp}\right)$ and $A_{\bar{J} U} V=A_{\bar{J} V} U$, then

$$
\begin{aligned}
\bar{R}(U, V, U, V)= & \hat{R}(U, V, U, V)-g\left(A_{\bar{J} U} V, A_{\bar{J} U} V\right)+g\left(A_{\bar{J} U} U, A_{\bar{J} V} V\right) \\
& +g\left(A_{h^{l}(U, U)} V, V\right)-g\left(A_{h^{l}(V, U)} U, V\right) .
\end{aligned}
$$

Now, let $W \in \Gamma\left(S\left(T M^{\perp}\right)\right)$ then for $U, V \in \Gamma\left(D^{\perp}\right)$, using (2.5), we have $g\left(A_{W} U, V\right)$ $=g\left(U, A_{W} V\right)$. Using this fact with $A_{\bar{J} U} V \in \Gamma\left(D^{\perp}\right)$, we get

$$
\begin{aligned}
g\left(A_{\bar{J} U} V, A_{\bar{J} U} V\right)-g\left(A_{\bar{J} U} U, A_{\bar{J} V} V\right) & =g\left(A_{\bar{J} V} U, A_{\bar{J} U} V\right)-g\left(A_{\bar{J} U} U, A_{\bar{J} V} V\right) \\
& =g\left(A_{\bar{J} U} A_{\bar{J} V} U, V\right)-g\left(A_{\bar{J} V} A_{\bar{J} U} U, V\right) \\
& =-g\left(\left[A_{\bar{J} V}, A_{\bar{J} U}\right] U, V\right) .
\end{aligned}
$$

On using (4.27) in (4.26), the assertion follows.

Now we define O'Neill's tensors [10] for a lightlike submersion. Let $\nabla$ be a connection of $M$ then tensors $\mathcal{T}$ and $\mathcal{A}$ of type $(1,2)$ are given by

$$
\mathcal{T}_{X} Y=\mathscr{H} \nabla_{\mathcal{V} X} \mathcal{V} Y+\mathcal{V} \nabla_{\mathcal{V}_{X}} \mathscr{H} Y, \mathscr{A}_{X} Y=\mathscr{H} \nabla_{\mathscr{H}_{X}} \mathcal{V} Y+\mathcal{V} \nabla_{\mathscr{H}_{X}} \mathscr{H} Y .
$$

Using (4.28), we have the following lemma.

Lemma 3. Let $\phi: M \rightarrow B$ be a lightlike submersion of a totally umbilical semitransversal lightlike submanifold of an indefinite Kaehler manifold $\bar{M}$ onto an indefinite almost Hermitian manifold $B$. Then we have the following:

(i) $\nabla_{U} V=\mathcal{T}_{U} V+\mathcal{V} \nabla_{U} V$.

(ii) $\nabla_{V} X=\mathscr{H} \nabla_{V} X+\mathcal{T}_{V} X$.

(iii) $\nabla_{X} V=\mathcal{A}_{X} V+\mathcal{V} \nabla_{X} V$.

(iv) $\nabla_{X} Y=\mathscr{H}_{X} Y+\mathcal{A}_{X} Y$,

for any $X, Y \in \mathscr{H}$ and $U, V \in \mathcal{V}$.

Theorem 10. Let $\phi: M \rightarrow B$ be a lightlike submersion of a totally umbilical semi-transversal lightlike submanifold of an indefinite Kaehler manifold $\bar{M}$ onto an indefinite almost Hermitian manifold $B$ such that $\bar{J}\left(D^{\perp}\right)=S\left(T M^{\perp}\right)$. Then $\bar{K}(X \wedge$ $V)=\left\|H^{s}\right\|^{2}-\left\|T_{X} V\right\|^{2}$, for any unit vector fields $X \in \Gamma(D)$ and $V \in \Gamma\left(D^{\perp}\right)$.

Proof. Let $X \in \Gamma(D)$ and $V \in \Gamma\left(D^{\perp}\right)$ then using the Theorem 5 and Lemma 3 with (4.3), we obtain

$$
\begin{aligned}
g(R(V, X) X, V)= & g\left(\nabla_{V} \mathscr{H}\left(\nabla_{X} X\right), V\right)-g\left(\nabla_{X} \mathscr{H}\left(\nabla_{V} X\right), V\right) \\
& -g\left(\nabla_{X} \mathcal{T}_{V} X, V\right)+g\left(\mathcal{T}_{[X, V]} X, V\right) .
\end{aligned}
$$

It should be noted that $g\left(\nabla_{V} \mathscr{H}\left(\nabla_{X} X\right), V\right)=-g\left(\mathscr{H}\left(\nabla_{X} X\right), \nabla_{V} V\right)$, and similarly $g\left(\nabla_{X} \mathscr{H}\left(\nabla_{V} X\right), V\right)=-g\left(\mathscr{H}\left(\nabla_{V} X\right), \nabla_{X} V\right)$. Therefore we have

$$
\begin{aligned}
g(R(V, X) X, V)= & -g\left(\mathscr{H}\left(\nabla_{X} X\right), \nabla_{V} V\right)+g\left(\mathscr{H}\left(\nabla_{V} X\right), \nabla_{X} V\right) \\
& -g\left(\nabla_{X} \mathcal{T}_{V} X, V\right)+g\left(\mathcal{T}_{[X, V]} X, V\right) .
\end{aligned}
$$


Since $\bar{J}\left(D^{\perp}\right)=S\left(T M^{\perp}\right)$ then using the Theorem 8 , we have $L(U, V)=0$, for $U, V \in \Gamma\left(D^{\perp}\right)$. Hence using the definition of $\mathcal{T}$ with (2.3) and (4.22), we get

$$
g\left(\mathcal{T}_{V} X, U\right)=-g\left(\mathcal{T}_{V} U, X\right)=-g(L(V, U), X)=0 .
$$

Now, using (4.22), we have

$$
g\left(\mathscr{H}\left(\nabla_{X} X\right), \nabla_{V} V\right)=g\left(\mathscr{H}\left(\nabla_{X} X\right), L(V, V)\right)=0 .
$$

Since $M$ is a totally umbilical then using (4.30), we obtain

$$
\begin{aligned}
g\left(\nabla_{X} \mathcal{T}_{V} X, V\right) & =-g\left(\mathcal{T}_{V} X, \bar{\nabla}_{X} V\right)=-g\left(\mathcal{T}_{V} X, \mathcal{V}\left(\nabla_{X} V\right)\right) \\
& =g\left(L\left(V, \mathcal{V}\left(\nabla_{X} V\right)\right), X\right)=0 .
\end{aligned}
$$

Since for a vertical vector field $V,[X, V]$ is always vertical therefore again using (4.30), we have

$$
g\left(\mathcal{T}_{[X, V]} X, V\right)=-g(L([X, V], V), X)=0 .
$$

Using (4.6) and (4.31)-(4.33) in (4.29), we obtain

$$
g(R(V, X) X, V)=g\left(T_{X} V, T_{X} V\right) .
$$

Since $M$ is a totally umbilical then using (2.6) and (4.34), we get

$$
\begin{aligned}
\bar{R}(X, V, X, V)= & -g\left(T_{X} V, T_{X} V\right)+g\left(h^{l}(X, X), \nabla_{V} V\right) \\
& +g\left(h^{s}(X, X), h^{s}(V, V)\right) .
\end{aligned}
$$

Now, using Kaehlerian property of $\bar{M}$, we have $\bar{\nabla}_{V} \bar{J} \xi=\bar{J} \bar{\nabla}_{V} \xi$, for $V \in \Gamma\left(D^{\perp}\right)$ and $\xi \in \Gamma(\operatorname{Rad}(T M))$. Using the Lemma 3 with (2.4) and then comparing the horizontal components of resulting equation, we obtain

$$
A_{\bar{J} \xi} V=-\bar{J} \mathcal{T}_{V} \xi
$$

Since $M$ is semi-transversal lightlike submanifold then for $\xi \in \Gamma(\operatorname{Rad}(T M)), \bar{J} \xi \in$ $\Gamma(\operatorname{ltr}(T M))$ and using (4.28) for any $U, V \in \mathcal{V}, \mathcal{T}_{U} V=\mathscr{H} \nabla_{\mathcal{V} U} \mathcal{V} V \in \mathcal{H}$. Therefore (4.36) implies that $A_{\bar{J} \xi} V \in \mathscr{H}$ or $A_{N} V \in \mathscr{H}$. Then for $V \in \Gamma\left(D^{\perp}\right)$ and $N \in$ $\Gamma(\operatorname{ltr}(T M))$, we have $g\left(\nabla_{V} V, N\right)=-g\left(V, \bar{\nabla}_{V} N\right)=g\left(V, A_{N} V\right)=0$. This implies that $\nabla_{V} V$ has no component in $\operatorname{Rad}(T M)$. Using this fact in (4.35) with (3.9), the assertion follows.

\section{REFERENCES}

[1] M. Barros and A. Romero, "Indefinite Kähler manifolds." Math. Ann., vol. 261, no. 1, pp. 55-62, 1982, doi: 10.1007/BF01456410

[2] A. Bejancu, "CR submanifolds of a Kaehler manifold. I." Proc. Amer. Math. Soc., vol. 69, pp. 135-142, 1978, doi: 10.2307/2043207.

[3] J.-P. Bourguignon and H. B. Lawson, "Stability and isolation phenomena for Yang-Mills fields." Commun. Math. Phys., vol. 79, no. 2, pp. 189-230, 1981, doi: 10.1007/BF01942061.

[4] J.-P. Bourguignon and H. B. Lawson, "A mathematician's visit to Kaluza-Klein theory.” Rend. Semin. Mat. Torino Fasc. Spec, vol. Special Issue, pp. 143-163, 1989. 
[5] K. L. Duggal and A. Bejancu, Lightlike submanifolds of semi-Riemannian manifolds and applications. The Netherlands: Kluwer Academic Publishers, 2013, vol. 364, doi: 10.1007/978-94017-2089-2.

[6] K. L. Duggal and D. H. Jin, "Totally umbilical lightlike submanifolds.” Kodai Math. J., vol. 26, no. 1, pp. 49-68, 2003, doi: 10.2996/kmj/1050496648.

[7] M. L. Falcitelli, S. Ianus, and A. M. Pastore, Riemannian submersions and related topics. Singapore: World Scientific, 2004.

[8] M. L. Falcitelli, S. Ianus, A. M. Pastore, and M. Visinescu, "Some applications of Riemannian submersions in physics." Rev. Roum. Phys., vol. 48, pp. 627-639, 2003.

[9] S. Kobayashi, "Submersions of CR submanifolds." Tohoku Math. J., vol. 39, no. 1, pp. 95-100, 1987, doi: $10.2748 / \mathrm{tmj} / 1178228372$.

[10] B. O'Neill, “The fundamental equations of a submersion.” Michigan Math. J., vol. 13, no. 4, pp. 459-469, 1966, doi: 10.1307/mmj/1028999604.

[11] B. O'Neill, Semi-Riemannian geometry with applications to relativity. New York: Academic press, 1983, vol. 103, doi: 10.1137/1028086.

[12] B. Sahin, "Transversal lightlike submanifolds of indefinite Kaehler manifolds." An. Univ. Vest Timis. Ser. Mat.-Inform, vol. 44, no. 1, pp. 119-145, 2006.

[13] B. Sahin, "On a submersion between Reinhart lightlike manifolds and semi-Riemannian manifolds." Mediterr. J. Math., vol. 5, no. 3, pp. 273-284, 2008, doi: 10.1007/s00009-008-0149-y.

[14] B. Sahin, "Riemannian submersions from almost Hermitian manifolds." Taiwanese J. Math., vol. 17, no. 2, pp. 629-659, 2013, doi: 10.11650/tjm.17.2013.2191.

[15] B. Sahin and Y. Gündüzalp, "Submersion from semi-Riemannian manifolds onto lightlike manifolds." Hacet. J. Math. Stat., vol. 39, no. 1, pp. 41-53, 2010.

[16] M. Vişinescu, "Space-time compactification induced by nonlinear sigma models, gauge fields and submersions." Czech. Journ. of Phys. B, vol. 37, no. 4, pp. 525-528, 1987, doi: 10.1007/BF01599959.

Authors' addresses

Rupali Kaushal

Punjabi University, Department of Mathematics, Patiala, Punjab, India.

E-mail address: rupalimathsepbi.ac.in

Rakesh Kumar

Punjabi University, Department of Basic \& Applied Sciences, Patiala, Punjab, India.

E-mail address: dr_rk37c@yahoo.co.in

Rakesh Kumar Nagaich

Punjabi University, Department of Mathematics, Patiala, Punjab, India.

E-mail address: nagaich58rakesh@gmail.com 